\title{
Designed Self Assembly of Nano-Liposomes in the Male Reproductive Tract for Model Drug Delivery to the Prostate
}

\author{
Sujoy K. Guha*, Vandana Chauhan and Shubhadeep Banerjee \\ School of Medical Science and Technology, Indian Institute of Technology, Kharagpur, 721302, India
}

\begin{abstract}
Using the compound Styrene maleic anhydride (SMA) as a model of a drug, a novel approach for sustained endogenously formed liposome encapsulated drug delivery to the prostate gland has been evolved. Intra vas deferens depot of a combination of high molecular weight SMA (SMAh, the drug) and low molecular weight SMA (SMAl) is formed by one time injection. The SMAh breaks down sperm membrane to provide from the sperms a continuous supply of phospholipids. The SMA1 forms cleavage centers causing slow breakaway of nano SMAh fragments. Vas peristaltic pressures provide the mechanical forces bringing reactants together leading to encapsulation of nano particles of SMAh within phospholipids cover giving a continuous supply of nano liposomes. A dimethyl sulphoxide (DMSO) constituent of the depot leads to sulfur attachment to the liposome on account of which by a prostate tissue sulfur affinity mechanism the nano liposomes traverse the vas deferens- prostate barrier. The concept has been tested by implantation of the SMA in the rat vas deferens. Formation of nano liposomes in vivo; actual encapsulation of SMA within the liposome; the overall drug Encapsulation Efficiency; presence of liposomes in the vas deferens fluid and transfer to the prostate have been confirmed by Transmission Electron Microscope (TEM) examination and Fluorescence microscopy following Nile Red staining of vas fluid and prostate tissue
\end{abstract}

Keywords: Styrene maleic anhydride, molecular weight, phospholipids, transport, vas deferens, prostate, liposome, sperm, electron microscopy, fluorescence microscopy.

\section{INTRODUCTION}

With increasing longevity greater numbers of males are coming into the age group of high incidence of prostate cancer. Therefore preventive measures are of major interest. An oral drug named finasteride taken regularly has shown promise. However oral administration has the inherent problem that a large amount of the drug needs to be ingested to get a small quantity to reach the prostate and the regular intake of large doses produces adverse side's effects. A more direct and short delivery route to the prostate is preferred. Blood vessels to the prostate gland are not accessible and sufficiently superficial to allow the use of drug containing skin patches for delivery. The other standard modes of intramuscular or intravascular administration are not acceptable for regular repeat administration. Therefore for the objective, it was necessary to explore new and unconventional pathways. In this endeavor the tube like structure, Vas deferens of the male reproductive tract which is the sperm transport duct was looked at as a potential candidate because it has anatomical relationship to the prostate gland. Two obstacles were confronted (i) that there is no known transport channel between the vas deferens and the prostate and (ii) the vas deferens has a thick wall and is normally not in close contact to the skin. Hence skin surface patch sustained drug delivery into the vas deferens duct is not feasible. The present research overcomes these hurdles by novel schemes [1]. Nano

*Address correspondence to this author at the School of Medical Science and Technology, Indian Institute of Technology, Kharagpur - 721 302, India; Tel: $91 \quad 99 \quad 33 \quad 897 \quad 285 \quad$ Fax: $91 \quad 3222 \quad 282221$; E-mail: guha_sk@yahoo.com liposomes are made to form inside the vas deferens and acquire a special character so that cross through the vas deferens-prostate barrier. Further, to achieve sustained delivery, a depot of the drug precursor is formed within the lumen of the vas deferens. The depot is such that (i) it interacts with the continuous supply of sperms to release from the sperm head lipids which are the w materials for liposome formation and (ii) breakdown very slowly to give a compound which has character of a drug and gets encircled by lipids to result in drug containing liposome. In this manner a continual formation of drug encapsulated liposome is obtained. Additionally the reactants have been so selected that the intra vas deferens oscillatory pressure resulting from the normal peristaltic contractions of the wall of the vas deferens [2] provides the necessary mechanical forces which are essential to liposome formation for bringing the reactant together [3, 4]. Liposomal drugs have been proven capable of changing the drug distribution in vivo, increasing the drug effectiveness and decreasing the drug toxicity [5].

For the research Styrene maleic anhydride (SMA) has been selected as the compound to serve as the "drug model" for demonstrating the two obstacle overcoming approach. Later when this novel concept is translated into clinical use appropriate cancer preventing drug which also has the special properties of releasing lipids from sperms and getting encapsulated within liposome will be used.

\section{MATERIALS AND METHODS}

To effect a vas-prostate transfer a specially engineered compound needs to be injected into the vas having an affinity to some particular entity of prostate tissue. In designing 
the molecule to be delivered several affinity entities were considered with a concurrent attempt to identify a matching molecule which would be biocompatible and of such a form that it could be injected into the lumen of the vas. The choice homed on to the "sulfur affinity" of epithelial prostate tissue. It is known that sulfur exhibits a high affinity for zinc [6] and the zinc present in the prostate tissue [7] gives it an affinity for sulfur. A molecule in the spermatic fluid with a sulfur moiety would tend to be picked up by prostate tissue. Yet sulfur affinity alone is not an adequate mechanism of transport. The vas deferens spermatic fluid contains sulfur containing amino acid cysteine [8] whereas this amino acid is not significantly present in the prostate. An additional "drive mechanism" need to be coupled to the sulfur affinity to effect a transport. In these respect liposomes, which are means of many targeted drug delivery schemes, provides features which may be utilized in the present tracer compound design. In fact liposome containing curcumin has been shown to enter prostate cancer cells in vitro. Liposome alone will not work for vas-prostate transfer. Hence combination of sulfur affinity and liposome was modality selected for the drug compound design.

The vas deferens injected male contraceptive RISUG $^{\circledR}$ which is a chemical complex of Styrene maleic anhydride (SMA) and Dimethyl sulfoxide (DMSO) has sulfur content. Also there is extensive background experience regarding administering this compound into the vas deferens [9]. Liposomes with encapsulated RISUG can be formed in vitro using the technique given by Bangham et al. [10]. But because the technique of formation of liposomes requires that all organic solvent such as DMSO be washed off there will be no sulfur on the surface of the liposome and all the sulfur of the RISUG will be trapped inside the lipid bilayer of the liposome. Therefore the sulfur affinity of the prostate cannot work on such in vitro prepared liposomes.

In vivo the vas lumen assembly of RISUG containing liposome was conceived (Fig. 1) based upon a novel formulation for which a patent application is pending [11] and that approach was adopted in the present study. The contraceptive RISUG is a SMA-DMSO polymer complex of long chain length and high molecular weight. This compound has been designed to be stable when implanted in the vas deferens and have spermolytic action for a long period of time extending to decade or more. For the purpose of forming liposomes in vivo a low molecular weight SMA-DMSO complex is mixed with the standard high molecular weight SMA-DMSO. The low molecular weight SMA-DMSO is relatively less stable and the breakup of this entity forms cleavage centers which cause the high molecular weight SMA-DMSO to shed nano particles of SMA-DMSO. Sperms flowing past the standard SMA-DMSO complex component undergo spermolysis [12] and release phospholipids from the sperm head. The phospholipids encapsulate the nano particles of high molecular weight SMA-DMSO to form liposomes. Also some of the nano particles attach to the exterior of the liposomes to provide the sulfur moiety. Since there is continuous supply of sperms, there is continuous spermolysis and a sustained supply of phospholipids. The breakdown of low molecular weight SMA-DMSO is also a continuing process and consequently so is the detachment of high molecular weight SMA-DMSO nano particles. Nano particles being of 30-60 nm in size a vas implant with $2 \mathrm{mg}$ of total SMA-DMSO complex can provide nano particles over a long period of time. Thus an implant of the high and low molecular weight combination in the vas deferens leads to sustained spermolysis and formation of liposomes RISUG containing liposomes with sulfur on its surface. This product meets the needs of being a vas to prostate transferable compound.

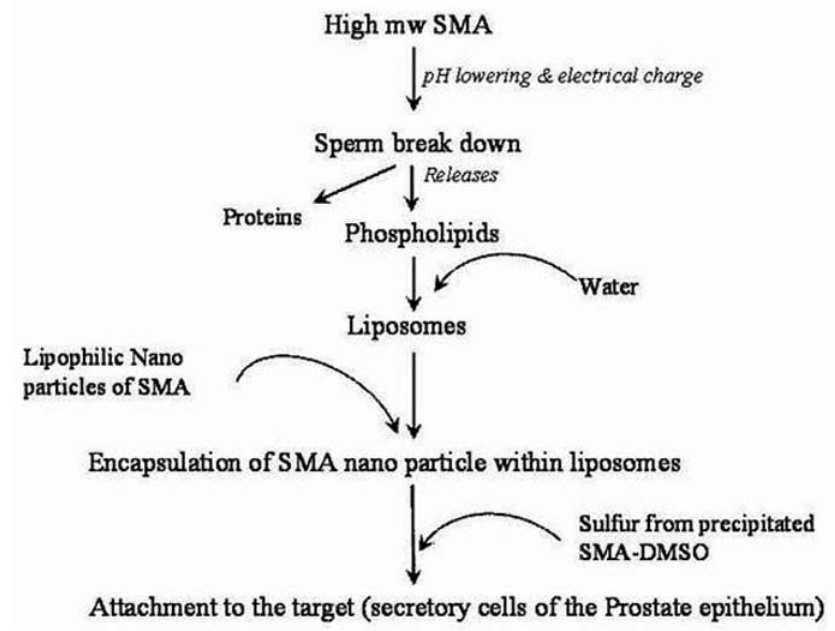

Fig. (1). Intra vas deferens liposome formation steps and transport to the prostate.

For the experimental study three groups of Wistar male albino rats of $150-200 \mathrm{~g}$ weight range with five rats in each group were taken. In the first group $5 \mu 1$ of DMSO was injected in one vas with the 26 gauge injection needle pointing towards the distal end of the vas deferens adopting a procedure described in an earlier publication from our group [13]. In the contralateral vas $5 \mu \mathrm{l}$ of DMSO complexed with $2 \mathrm{mg}$ of the high plus low molecular weight SMA was injected. After an interval of 3 weeks rats were sacrificed with an overdose of ether anesthesia and by surgical exploration the terminal ends of both vas near the ampulla of the vas was exposed and sectioned. The spermatic fluid from each vas deferens was squeezed out separately. Both the sample fluid were suspended separately in HEPES buffer and centrifuged. The pellet was resuspended in $0.1 \mathrm{M}$ cacodylate buffer containing $2.5 \%$ glutaraldehyde. Samples were kept at $4^{\circ} \mathrm{c}$ for 4 hrs. Thereafter preparations were centrifuged and pellets resuspended in HEPES buffer. From the top layer liquid with particles contents was drawn. Negative staining was performed with $1 \%$ ammonium molybdate solution and examined with a Transmission Electron Microscope. (JEOL JEM2100 High Resolution Transmission Electron Microscope)

In the second group of rats $2 \mathrm{mg}$ of the high plus low molecular weight SMA in $5 \mu \mathrm{l}$ of DMSO was injected into both vasa of each rat. After three weeks the animals were sacrificed and the spermatic fluid from both vasa was squeezed out and mixed with HEPES buffer. A drop of the mixture was taken on a glass slide and the drop was covered with Nile Red Fluorescent stain. The preparation was examined in a Fluorescent Microscope in the light wavelength band of $485-525 \mathrm{~nm}$.

Both vasa in the third group of rats were injected with 2 $\mathrm{mg}$ of the high plus low molecular weight SMA in $5 \mu 1$ of 


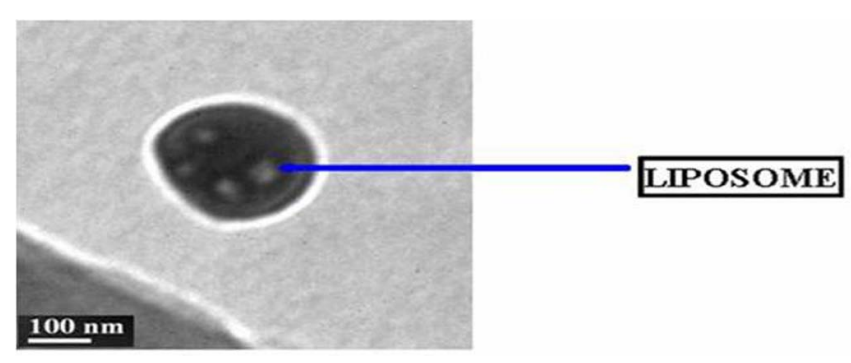

Fig. (2). TEM image of liposome within vas deferens.

DMSO. After three weeks the rat were sacrificed with an ether overdose of ether anesthesia and the prostate gland was dissected out. Afterwards the prostate gland was cut into small pieces and was kept in collagenase $(2 \mathrm{mg} / \mathrm{ml})$ dissociation solution at $37^{\circ} \mathrm{c}$ for $1 \mathrm{hr}$. Centrifugation was done at $30 \mathrm{~g}$ for $3 \mathrm{~min}$. Fluorescent staining was performed with Nile Red fluorescent marker dye and the preparation examined in a Fluorescent Microscope in the light wavelength band of 485$525 \mathrm{~nm}$.

\section{Quantum of Entrapment of SMA in the Formed Liposomes}

The liposomes formed after the sperms came in contact with the styrene maleic anhydride - dimethyl sulfoxide complex injected into the vas of the rats was checked for the entrapment of the drug within the liposomes. Into each vas deferens of a rat $5 \mathrm{mg}$ of SMA mixed with DMSO was administered. By observation it was confirmed that over a period of one week there was no spontaneous ejaculation. At the end of the week the entire vas fluid from both vas of each rat was collected. The vas fluid was filtered through a 0.2 micron membrane filter to remove any debris. The fluid was then centrifuged with an eppendorf Centrifuge $(11,000 * \mathrm{~g}$, 20 mins) through Microcon YM-10 centrifugal filter devices (Millipore) with a cutoff value of $10 \mathrm{kDa}$ to separate and concentrate molecules with molecular weight greater than $10 \mathrm{kDa}$. Methanol was added to the obtained retentate and was then severely vortexed to break down the liposomes and release the encapsulated SMA. The amount of SMA present in the solution, which is the SMA released from the liposomes and was determined by spectrophotometer at the wavelength $260 \mathrm{~nm}$ and then relating it to the standard curve for the drug. The experiment was repeated in one more rat.

\section{RESULTS}

The findings confirm that SMA is entrapped within liposomes. The amount of SMA present in the liposomes formed over one week was $0.08 \mathrm{mg}$ in one rat and $0.11 \mathrm{mg}$ in the other rat with an average of $0.098 \mathrm{mg}$. That is out of the total amount of SMA formed as a depot approximately 1 $\%$ is present in liposomes formed in a period of one week following SMA depot formation. SMA being an amphiphilic molecule it is entrapped within the liposomes with its aromatic ring in contact with the backbone of the phospholipids molecules and the hydrophilic region of the drug interacts with the head group region of the phospholipids molecules.

TEM examination of the vasa fluid from the SMA injected vas deferens of rats in the first group showed distinct forms as shown in which are characteristic of liposomes (Fig. 2). Some of the liposomes are in clusters. Size of the liposomes varies from 30 to $300 \mathrm{~nm}$. When the liposomes are collected from the top layer of the final resuspended pellet in the TEM examination sample preparation procedure the smaller liposomes show up more in the TEM image. No liposome like forms was seen in the specimen of the vas fluid taken from the side in which only DMSO was injected.

Fluorescent microscopy of vas fluid drawn from animals of the second group showed circular forms with lesser stained centers which is a characteristic of liposomes (Fig. 3). Such fluorescence microscopic images of liposomes are already cited in many research articles previously $[14,15]$. Additionally the image (Fig. 4) shows spermolytic action both on the head and tail of the sperms. The spermolysis is with the same character as evidenced in earlier RISUG study [16].

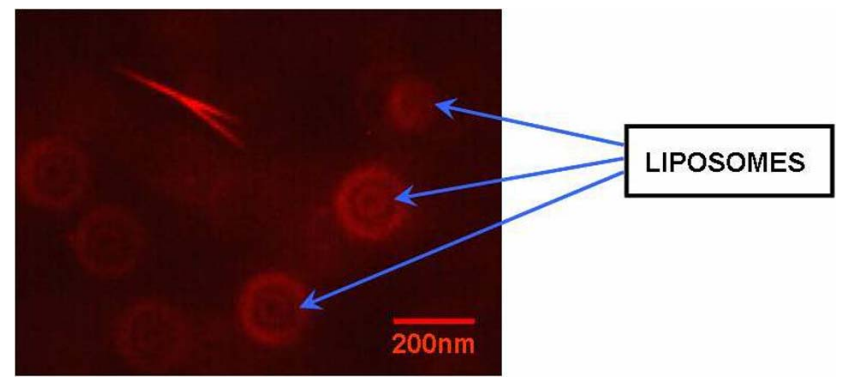

Fig. (3). Fluorescence microscopy image of liposome within vas deferens.

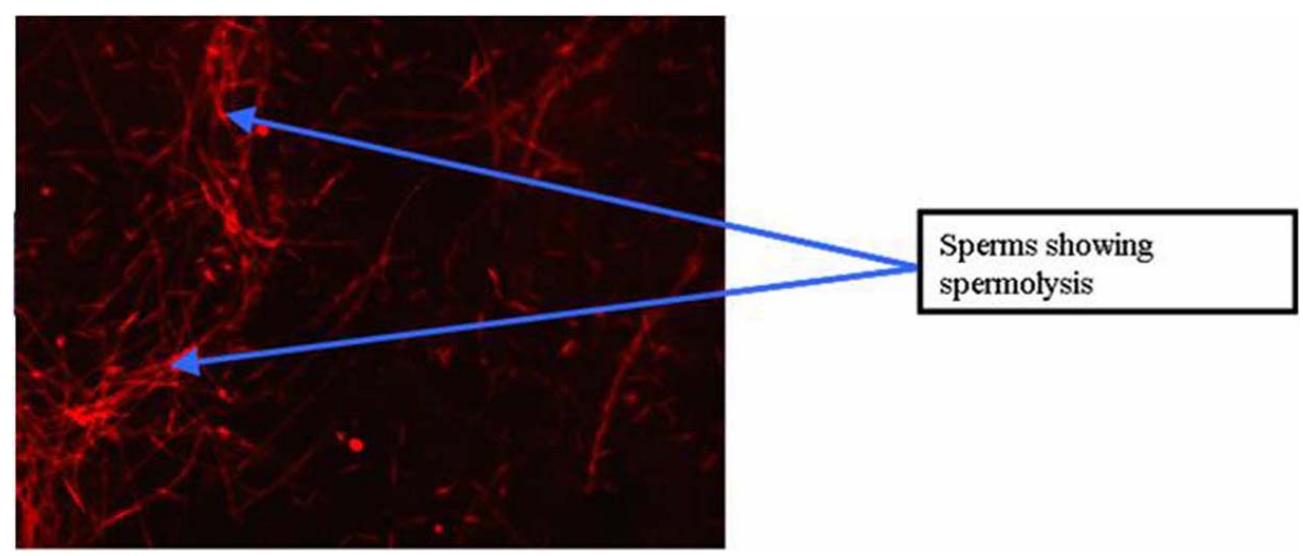

Fig. (4). Fluorescence microscopy image of sperms showing spermolysis. 


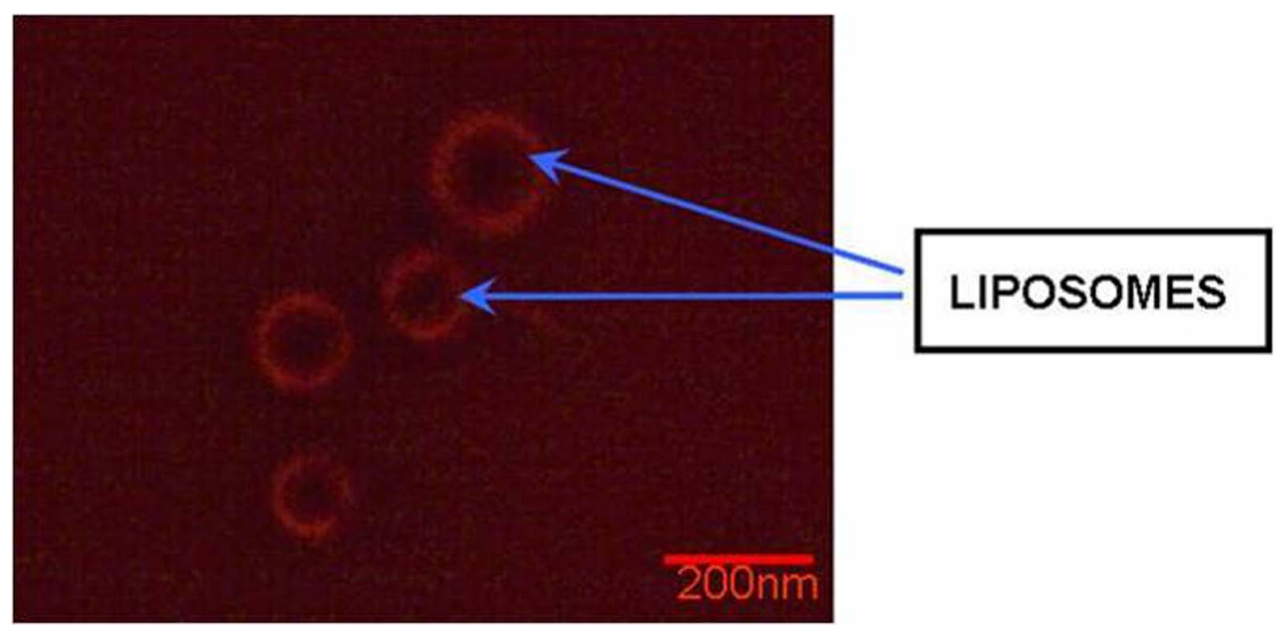

Fig. (5). Fluorescence microscopy image of liposome in prostate gland.

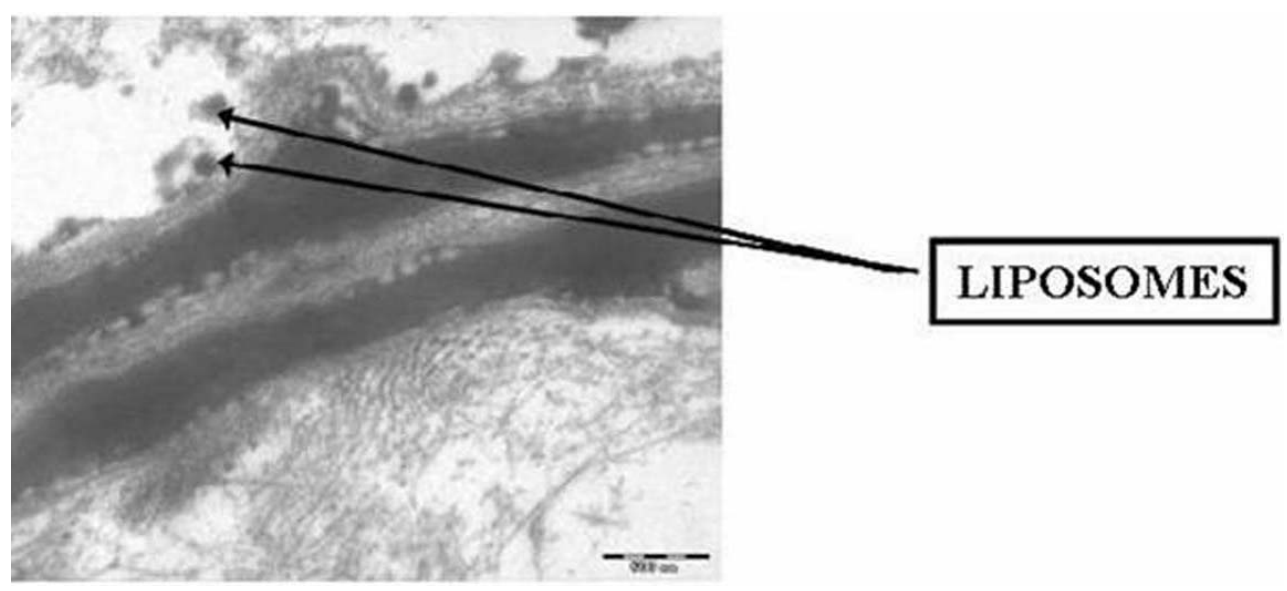

Fig. (6). TEM image of liposome in the epithelial region of prostate.

Under fluorescent microscopy and TEM liposomes were also detected in prostate gland tissue of rats of the third group. The form of the liposomes seen was similar to that observed in the vas fluid of the second group of rats (Figs. 5 and 6). In a separate control experiment it was noted that prostate tissue of rats which have been given a DMSO injection into the vas deferens do not show the presence of any forms which are similar to the liposomes seen in the studies on the three experimental group of animals.

\section{DISCUSSION}

The data from the study on the first group of animals indicates that injection of the combination of High and low molecular weight SMA complexed with DMSO leads to the formation of liposomes within the vas deferens. Administration of DMSO alone is not adequate for the formation of liposomes. The formation of liposomes is further confirmed by an independent technique of fluorescent microscopy in the study on the second group of rats. The presence of liposomes in the prostate of the third experimental group demonstrates a transfer of the liposomes formed in the vas deferens to the prostate tissue.

The results may be interpreted to conclude that there does exist a mechanism by which a structural form greater than of nano dimension can enter into prostate tissue from the vas deferens. This route may be termed as a "channel" recognizing that it is a biochemical channel rather than a morphologi- cal channel. Whether the transport is a passive transport or an active transport cannot be determined from the present data. The fact that sulfur affinity is an important aspect of the transport mechanism would point to an active transport. Further research is required to estimate the channel capacity that is the maximum size of liposomes that can be transported and the number that can be moved per unit time.

By means of the approach presented a continuous supply of liposome entrapped drug can be produced within the vas deferens and some of it enters the prostate. The placement of small molecular weight SMA cleavage centers and consequent breakaway of nano particles of high molecular weight SMA leads to a progressive diminution of the SMA in the depot. The supply of sperms is abundant and therefore the supply of phospholipids is not a limiting factor to the liposome formation. If all the SMA released is entrapped and the SMA entrapment is $1 \%$ of the total depot per week then the depot will suffice for continuous supply of SMA containing liposomes for 100 weeks that is approximately 2 years which is the usual lifetime of a rat. There is likely to be some SMA released which is not entrapped in liposomes and is lost in free form. This factor reduces the life span of the depot. There is always a compensating phenomenon that the release from a depot declines gradually following the initial creation of the depot. In overall it can be expected that the depot will serve for a life time. In case extended studies show that the life span of the depot is falling short of the 
requirement the quantum of low molecular weight SMA as cleavage centers can be reduced.

The method of formation of the liposomes in vivo opens up the possibility of assembly of other compounds as drugs into the liposome form within the vas deferens and delivery to the prostate. The vas deferens can then be a drug assembly and delivery pathway.

\section{ACKNOWLEDGEMENT}

Research supported by a grant from the Government of India, Ministry of Health and Family Welfare.

\section{REFERENCES}

Guha, S.K. Styrene maleic anhydride based formulation for male contraception and prostate cancer. World Intellectual Property Organization, Patent Cooperation Treaty (PCT), International Publication Number WO2009/113108 A2, September17, 2009.

[2] Guha, S.K.; Kaur, H.; Ahmed, H. Mechanics of fluid transport in the vas deferens. Med. Biol. Eng., 1975, 13, 518.

[3] Khan, D.R.; Rezler. E.M.; Lauer-Fields, J.; Fields, G.B. Effects of Drug Hydrophobicity on Liposomal Stability: Chem. Biol. Drug Des., 2008, 71, 3 .

[4] Stensrud, G.; Sande, S.A.; Kristensen, S.; Smistad, G. Formulation and characterization of primaquine loaded liposomes prepared by a $\mathrm{pH}$ gradient using experimental design. Int. J. Pharm., 2000, 198, 213.

[5] Pan, X.Q.; Jones, S.; Cox, K. The way that PEGyl-DSPC liposomal doxorubicin particles penetrate into solid tumor tissue. Drug Target Insights, 2006, 1, 29.
[6] Wolfgang, M. Zinc and sulfur: A critical biological partnership. Biochemistry, 2004, 43, 3301.

[7] Huang, L.; Kirschke, C.P.; Zhang, Y. Decreased intracellular zinc in human tumorigenic prostate epithelial cells: a possible role in prostate cancer progression. Cancer Cell Int., 2006, 6,10 .

[8] Udby, L.; Bjartell, A.; Malm, J.; Egesten, A.; Lundwall, A.; Cowland, J.B. Characterization and localization of cysteine-rich secretory protein 3 (CRISP-3) in the human male reproductive tract. $J$. Androl., 2005, 26, 333.

[9] Guha, S.K.; Singh, G.; Srivastava, A.; Das, H.C.; Bhardwaj, J.C.; Mathur, V.; Das, S.K. Two year clinical efficacy trial with dose variations of a vas deferens injectable contraceptive for the male. Contraception, 1998, 58, 165.

[10] Bangham, A.D.; Standish, M.M..; Watkins, J.C. Diffusion of univalent ions across the lamella of swollen phospholipids. J. Mol. Biol., $\mathbf{1 9 6 5}, 13,238$

[11] Guha, S.K. Styrene maleic anhydride based formulation for male contraception and prostate cancer. Indian Patent Journal, 19, 20096 , 2009.

[12] Guha, S.K. Biophysical mechanism-mediated time-dependent effect on sperm of human and monkey vas implanted polyelectrolyte contraceptive. Asian J. Androl., 2007, 9, 221.

[13] Misro, M.M.; Guha, S.K.; Singh, H.; Mahajan, S.; Ray, A.R.; Vasudevan, P. Injectable non-occlusive chemical concentration in male. Contraception, 1979, 20, 467

[14] Janas, T.; Yarus, M. Visualization of membrane RNAs. RNA, 2003, 9,1353 .

[15] Muller, W.; Koynov, K.; Fischer, K.; Hartmann, S.; Pierrat, S.; Basche, T.; Maskos, M. Hydrophobic shell loading of PB-b-PEO vesicles. Macromolecules, 2009, 42, 357.

[16] Guha, S.K. Bioengineering in Reproductive Medicine. CRC Press, Inc., 1990.

(C) Guha et al.; Licensee Bentham Open .

This is an open access article licensed under the terms of the Creative Commons Attribution Non-Commercial License (http://creativecommons.org/licenses/by-nc/3.0/) which permits unrestricted, non-commercial use, distribution and reproduction in any medium, provided the work is properly cited. 